\title{
LVI. On thunderstorm electricity
}

\section{C.T.R. Wilson M.A. F.R.S.}

To cite this article: C.T.R. Wilson M.A. F.R.S. (1909) LVI. On thunderstorm electricity , Philosophical Magazine Series 6, 17:100, 634-641, DOI: 10.1080/14786440408636636

To link to this article: http://dx.doi.org/10.1080/14786440408636636

册 Published online: 21 Apr 2009.

Submit your article to this journal $\pi$

Џll Article views: 6

Q View related articles $\asymp$

4 Citing articles: 4 View citing articles 주다. 
layer, and, what is still more important, the cloud itself from base to summit during the time that the lightning-discharges were taking place was never 2,500 metres in height: thus supersaturation to a fourfold degree could not possibly have taken place.

To sum up the discussion, we may state that the following six reasons have been given for considering the theory, first proposed by Wilson, and subsequently expanded by Gerdien, unsatisfactory as an explanation of the electrical effects connected with thunderstorms.

\section{A. Electrical.}

(1) The theory does not account for the frequent lightning discharges observed during thunderstorms.

(2) It cannot account for the large charges of electricity carried down by rain during thunderstorms.

(3) It is extremely improbable that any electrical separation would take place as the result of water vapour being condensed from fourfold supersaturated air onto negative ions.

\section{B. Meteorological.}

(4) An extensive stratum of supersaturated air could not form above an ascending eurrent.

(5) Even if such a stratum were once formed it could not exist after precipitation commenced from the condensation layer above it.

(6) The meteorological phenomena observed during thunderstorms do not lend any support to the theory.

Simla, Nov. 11, 1908.

LVI. On Thunderstorm Electricity.

$B y$ C. T. R. Wilson, M.A., F.R.S.*

MR. SIMPSON has been kind enough to send me a 11 copy of his paper "On the Wilson-Gerdien Theory of Thunderstorm Electricity."

I should like at the outset to remark that I have nowhere myself attempted to account for the strong electric fields of thunderstorms by the theory which Mr. Simpson attributes to me. I have merely regarded condensation on negative ions as one of the possible factors in the production of such fields.

* Communicated by the Author. 
The letter to 'Nature' from which he quotes was in defence of the view (first suggested by J. J. Thomson) that the positive charge of the atmosphere may be due to a preponderance of negative ions being carried down in rain, in consequence of their efficiency as condensation nuclei being greater than that of the positive ions.

There ean, I think, be no doubt that in fine weather regions there is a positive current downwards from the atmosphere into the ground in accordance with the direction of the electrical field. The current per sq. $\mathrm{cm}$. of the ground can in fact be measured indirectly from the potential gradient and conducting power of the air, as in the experiments of Gerdien and others, or more directly as in my own experiments (Proc. Roy. Soc. vol. lxxx. p. 537, 1908); and the measurements by different methods give values which agree. Until there is evidence to the contrary, it is natural to assume that in wet weather regions a compensating process is going on, due to a preponderance of negative electricity being carried down by rain, the electrical current in the atmosphere from the regions of precipitation to the fine weather regions being one of conduction (Proc. Camb. Phil. Soc. vol. xiii. p. 363) in the upper layers. It may eventually turn out that there is not the required excess of negatively charged rain, and that we shall be compelled to look to some other, possibly cosmical, source for the supply of negative electricity.

Both Wlster and Geitel, and also Gerdien, have, however, found such an excess of negatively charged rain. If further investigations should confirm the apparent preponderance of negative rain found in those earlier experiments, then the superior efficiency of the negative ion as a nucleus may, I think, be looked upon as one possible factor in bringing about this preponderance. It is unfortunately exceedingly difficult in measurements of the charge carried down by rain in heavy showers and thunderstorms to avoid spurious effects.

In the only paper where I have discussed the possible factors causing strong electrical fields accompanying heavy showers or thunderstorms ('Nature,' vol. lxviii. p. 102, 1903), I pointed out that the difference between positive and negative ions will not of itself account for intense fields. I should like to quote the passage-

"The following are possible factors in the production of the intense electrical fields which accompany havy showers-

"A less degree of supersaturation is required to make water condense on the negative than on the positive ions (C. T. R. Wilson, Phil. Trans. vol. cxciii. p. 289). Thus if condensation takes place from the supersaturated condition, the drops 
formed are likely to be negatively charged; that the drops, formed in ionized air by expansions slightly exceeding that required to cause condensation on negative ions, are actually negatively charged has been proved by H. A. Wilson (Phil. Mag. April 1903). Since, however, each drop will only carry the very small ionic charge, the electrical effect will be small if only a few large drops are formed; if a large number of negative ions serve as nuclei of condensation, the drops will be small, and will only fall slowly relatively to the air ; the resulting electric field cannot exceed that which drives positive ions downwards as fast as the negatively charged drops fall under the action of gravity. The field initially produced may, however, be strong enough to induce coalescence of drops which come in contact (Lord Rayleigh, Roy. Soc. Proc. vol. xxviii. p. 406), and we may this get drops carrying many times the charge of one ion, and large enongh to fall rapidly. Strong tields may then result.

Again, we should expect (Nature, vol. lxii. p. 149) drops falling through ionized air to become negatively charged as a result of the difference in the mobility of the positive and negative ions. This effect has in fact been experimentally demonstrated by Schmanss (Ann. d. Physik, vol. ix. p. 224).

If collisions resulting in splashing occur between raindrops (and they are likely to be frequent in the up-rush of air in thunderstorms) positiveily charged rain may be formed. For, as Lenard has shown, when splashing of pure water occurs, as for example in waterfalls, the air in the neighbourhood acquires a negative, the water a positive charge.

Apart from the Lenard effect, the splashing resulting from the collision of drops in an electric field may have large effects, either in intensifying or diminishing the electric fields already existing, the action being like that of an electrostatic influence-machine. The result would be to increasethe intensity of the field if the splashes were thrown out from the lower portion of the combined drop. If, for example, the field were such as to produce positive electrification on the lower surface of a neutral drop, a droplet leaving the lower surface would be positively charged, and being carried upwards by the air relatively to the large drop, would add to the intensity of the primary field."

The difficulty mentioned in the first paragraph of the passage quoted is in itself sufficient to make untenable the theory of thunderstorms which Mr. Simpson criticises. Taking the example given by him and assuming a cloud layer of continually increasing thickness to be formed, we can readily see that unless larger drops are produced by 
coalescence, the maximum strength of field could not exceed one volt per centimetre; for in stronger fields the positive ions would travel downwards relatively to the air faster than the drops.

Small uncharged drops do not generally coalesce on coming in contact, but, as Lord Rayleigh has shown, electrification of the drops causes coalescence, and indeed he has suggested that the large drops of thunderstorms may be due to this cause. In his experiments, it is true, the conditions determining coalescence were such that the coalescing drops carried a charge of electricity while the drops condensed on the ions have a charge which is probably too small to have any effect of this kind. But there is little doubt that coalescence would also take place between neutral drops in an electric field, i.e. between drops of which the impinging suriaces were oppositely charged by induction. Experiments. could alone decide what strength of field would be required to cause coalescence.

We should not necessarily get an upper cloud of more than very small vertical thickness, even if the upward air-current were sufficient to support the drops when first condensed on the negative ions from the supersaturated vapour.

For the critical supersaturation ( $i$. $e$. the supersaturation necessary to cause condensation on the negative ions) will be reached at a level which will be continually rising. The critical supersaturation will be attained by a given portion of the air when it has risen to a definite height, not above the present position of the upper surface of the lower cloud, but above the level which that upper surface had when the portion of air under consideration escaped from it. The level of critical supersaturation would then in the absence of condensation travel upwards with a velocity depending upon that of the upper surface of the lower cloud.

The size of the drops which separate out when the critical supersaturation is first reached depends of course on the number of negative ions present, and can be calculated for given conditions. If these initially formed drops are sufficiently small to be carried upwards faster than the upward velocity of the level of critical supersaturation (which we may call the critical velocity), then a layer of cloud will be formed continually increasing in tuickness by the condensation of drops at this level of critical superaturation. Ice-particles would be specially likely to be carried upwards with more than the critical velocity. If, however, the drops condensed on the negative ions are large enough to be carried up with less than the critical velocity, the critical supersaturation 
will never be attained below the first-formed drops, and the rising air will remain supersaturated to nearly the critical extent till it reaches these drops. They will thus grow extremely rapidly until large enough to fall through the supersaturated layer and lower cloud.

The supersaturated layer above the top of a cumulus cloud which is rapidly developing into cumulo-nimbus would probably in most cases be of quite small vertical thickness. Under the condilions just considered we might expect a thin cloud-cap to be formed suddenly over the head of the cumulus and sink rapidly into it. (I have on several occasions observed something very like this taking place; but it is of course very easy to be deceived in such matters.) It is possible that a process of this kind would determine the transition from cumulus to cumulo-nimbus. The drops formed on the ions may themselves fall through the lower cloud and reach the ground as rain. At the same time the electric field between the free positive ions above and the negatively charged drops will by the fall of the latter be extended through the upper part of the lower cloud. This field may help coalescence both between droplets of the lower cloud and between them and the larger drops which have come from above it. When this stage has been reached the effect of splashing referred to in the last paragraph quoted above from 'Nature' may be expected to become effective. It would be interesting to know from direct experiment what is the nature of the splash occurring when a large falling drop overtakes a smaller one. It seems not unlikely, however, in the light of Worthington and Cole's photographs of the "splash of a drop " falling into a vessel of water (Phil. Trans. vol. clxxxix. A. p. 137, 1897), that droplets may be thrown out from the underside of the combined drop if coalescence takes place. If we have a vertical electrical field already in existence the splashing process will in that case continually add to the intensity of the field.

It is in such ways that I think condensation upon negative ions may have to be taken into account in relation to thunderstorms; the first onset of the rain may be due to this cause, and it may also determine the direction of the initial electric field. The intense electrical fields of thunderstorms and the large charges carried down by the drops to the ground are possibly due to electrostatic induction effects accompanying collisions of the drops ; the prevailing sign of the field and of the charge carried by the drops may be determined by the initial condensation on negative ions.

Elster and Geitel long ago suggested (Wied. Ann. vol. xxv. 
p. 121,1885$)$ that the intense electric fields of thunderstorms might be due to electrostatic influence effects accompanying the fall of larger drops through a clond of smaller ones-a weak initial electrical field being assumed.

To what extent condensation upon the ions is likely to be effective after the initial stages of the showers is a difficult question into which I do not propose to enter. I have never regarded it as at all likely that the supersaturation necessary to cause condensation upon the positive ions could ever occur in the atmosphere, as Elster and Geitel and others have imagined.

The question of the occurrence of supersaturation in the atmosphere is of course fundamental in relation to the question under discussion. In the article in 'Nature' quoted by Mr. Simpson nothing was said about the condition of the atmosphere immediately above the cloud. The argument was intended to show how, even if the lower moisture-charged layers were highly charged with dust-particles, a supersaturated layer might still result, and the upper atmosphere remain dust free.

To make the matter clearer, let us assume that above a lower dust- and moisture-charged layer we have a dry and dust-free layer. (It may be remarked that a large mass of air in the atmosphere can hardly have become dry otherwise than by the condensation and subsequent precipitation of the water from it-a process which is likely to remove from it the dust-particles as well. It is of course possible that there may be in the upper atmosphere a continual production of dust-particles-under which title we may include all persistent nuclei more efficient than the ordinary negative ion.) If we imagine a local ascensional current to begin, the surface of separation of the two layers will rise; and when it has reached the level corresponding to saturation for the lower layer a cumulus cloud will begin to form. The upper surface of this cumulus cloud marks appoximately the surface of separation between the dry and moist layers. The transition from the dry to the moist layer cannot be absolutely sudden on account of diffusion; in consequence condensation will first begin somewhat below the upper limit of the dustcharged air. As the air continues to rise the cloud will thus at first grow partly by additions to its upper edge, the newest and smallest drops being at the top. If the transition layer between the dry and moist air continues to ascend, a stage will be reached when saturation extends to the highest level reached by the dust-particles. Beyond this stage the cloud will cease to grow by addition of new drops at its upper 
surface, and a super-saturated layer will begin to form above it.

Although I have never observed the peculiar formation described by $\mathrm{Mr}$. Simpson as being required for thunderclouds according to the theory he is criticising, I see no reason on that account to conelude that condensation on negative ions may not take place in the atmosphere. If the supersaturated layer be thin, the condensation on the negative ions will not in itself be at all a conspicuous phenomenon although its effects may be by no means unimportant. It is possible, however, that more striking phenomena may also be the result of condensation on the negative ions. In a typical fully developed cumulo-nimbus clond, while the lower part of the cloud has the appearance of ordinary cumulus, the upper part has an entirely different appearance, of which there are several different varieties. In some cases the appearance is suggestive of rain or snow falling from some height above the lower cumuliform portion of the cloud. It is possible that this upper portion of the cumulo-nimbus (the false cirrus) may be due to condensation on the negative ions. Until more has been done by experiment and observation to throw light on the processes going on in clonds, it hardly seems profitable to consider in detail the possible cloud forms that might be expected to result from condensation on negative ions.

There is one statement in Mr. Simpson's paper which, while not seriously affecting his argument, appears to call for remark. In discussing the effect of a lightning-flash he says:- "Now Barus has shown that whenever ions are formed in dust-free air there is produced at the same time a large number of nuclei which exist for a long time after the ions have disappeared. As water-vapour is very readily deposited on the nuclei, fourfold supersaturation is prevented in air in which they are present unless the supersaturation is caused by exceedingly rapid rarefaction." It is quite true that spark-discharges in air, as well as other ionizing processes which are accompanied by chemical effects, do produce large and persistent nuclei in addition to the ordinary ions ; a lightning-flash will therefore almost certainly produce such nuclei. But the ordinary ionizing radiations when of moderate intensity do not produce, in uncontaminated air, nuclei more efficient than the negative ions.

I shall conclude with the following three statements expressing summarily my views on the questions raised by Mr. Simpson.

1. I have never regarded condensation on the ions as in 
itself a sufficient cause for the strong electrical fields of thunderstorms.

2. If, however, we assume the occurrence of supersaturation in the atmosphere, condensation on the negative ions is likely to be of importance in connexion with the production of. precipitation, and as tending to cause a preponderance of negatively charged rain to be carried down to the earth.

3. The question of the occurrence of supersaturation in the atmosphere must still, I think, be regarded as an open one.

LVII. On the Faraday-Maxwell Mechanical Stress ; and on Atherial Stress and Momentum in general. By C. V. Burton, D.Sc.*

1. LTHOUGH the more recent development of elecA trical theory has shown the difficulty of accepting as anything more than an analogy the Faraday-Maxwe!l "tension along the lines of force and equal pressure across them," this specification of rtherial stress must always be regarded as one of the most profound and important contributions ever made to the progress of physical science. Maxwell's analysis from which this mechanical retherial stress was deduced $t$, and which gave a precise form to Faraday's theory, was probably as cogent in directing attention to the possibilities of an "intervening medium" as was his great and enduring system of electromagnetic equations. As the merely analogical significance of Maxwell's "mechanical stress" does not seem to be quite generally appreciated - that specification of stress being still spoken of by physicists of distinction as if it necessarily represented the actual stress in the medium - the brief exposition which follows is perhaps not wholly uncalled for.

2. For an electrostatic field in free æther, the FaradayMaxwell mechanical stress at any point is a tension $\mathrm{K}^{2} / 8 \pi$ along the lines of force, with a pressure of like magnitude in all directions perpendicular to the lines of force; $\mathrm{R}$ being, in electrostatic measure, the electromotive intensity at the point in question. To the student of twenty odd years ago this stress was certainly a stumbling-block; for in a medium whose electromagnetic properties are expressible by a system of linear equations, and in which electromagnetic disturbances are propagated with a velocity independent of amplitude and of wave-length, it is not easy to realize why any

* Communicated by the Author.

+ 'Treatise,' vol. i. chap. v.

Plit. Mag. S. 6. Vol. 17. No. 100. April 1909. 2 X 